\title{
Incidence and factors influencing on Helicobacter pylori infection recurrence
}

\author{
B. J. Gómez Rodríguez, M. Rojas Feria, M. J. García Montes, R. Romero Castro, P. Hergueta Delgado, \\ F. J. Pellicer Bautista and J. M. Herrerías Gutiérrez
}

Service of Digestive Diseases. Hospital Universitario Virgen Macarena. Sevilla. Spain

Gómez Rodríguez BJ, Rojas Feria M, García Montes MJ, Romero Castro R, Hergueta Delgado P, Pellicer Bautista FJ, Herrerías Gutiérrez JM. Incidence and factors influencing on $\mathrm{He}$ licobacter pylori infection recurrence. Rev Esp Enferm Dig 2004; 96: 620-627. urea breath test should be performed to detect reinfection in order to avoid ulcer complications.

Key words: Reinfection. Recrudescence. Recurrence. Eradication. Helicobacter pylori. Urea breath test.

\begin{abstract}
Objectives: a) to determine the rate of $H$. pylori reinfection after successful eradication in a 4-year follow-up study; and b) to evaluate the contribution of different factors in the infection relapse.

Patients and methods: a total of 208 patients (age range 18-81 years; average 50 years; 87 women) who had been successfully treated for $H$. pylori infection were included. Annually, urea breath test was assessed to determine $H$. pylori status after eradication. Age, sex, rural/urban environment, smoking habit, treatment regimens against $H$. pylori and urea breath test values were evaluated.

Results: reinfection occurred in $9,6 \%$ of patients observed, $6,7 \%(14 / 208)$ in the first year, $1,9 \%(4 / 208)$ in the second year, $1 \%(2 / 208)$ in the third and $0 \%$ in the forth. Risk factors for infection recurrence were younger age and higher values of urea breath test in the multivariante analysis.

Conclusions: annual reinfection rate was 2,4 pacients-year. Younger patients and higher values of urea breath test were factors associated with a higher rate of reinfection relapse. Annually,
\end{abstract}

Recibido: 26-08-03

Aceptado: 10-02-04.

Correspondencia: Blas José Gómez Rodríguez. Servicio de Aparato Digestivo. Hospital Universitario Virgen Macarena. Avda. Doctor Fedriani, 3. 41071 Sevilla . Telf.: 955008 801. Fax: 955008 805. e-mail: jmhg@us.es.

Trabajo con soporte financiero mediante: Accésit al Premio al Mejor Proyecto de Investigación titulado: "Reinfección por Helicobacter pylori", otorgado en el año 2001 por la Sociedad Andaluza de Patología Digestiva. Premio para Proyecto de Investigación titulado: "Reinfección por Helicobacter pylori", otorgado en el año 2002 por la Fundación Española de Patología Digestiva.

\section{INTRODUCTION}

Helicobacter pylori infection is an essential factor in the pathogenesis of peptic ulcer disease, MALT lymphoma and gastric adenocarcinoma. Eradication heals active ulcers, and in the long term reduces ulcer relapse and complications (1).

There are two types of recurrence of $H$. pylori infection after successful treatment: recrudescence and true reinfection. Recrudescence is defined as a relapse of previous infection, whereas true reinfection is an infection with a new strain that is different from the former $(2,3)$.

Reinfection rate is around $6.25 \%$ in some parts of Asia (4) and $2.4 \%$ in India (5). In developed countries the reinfection rate is lower than $1 \%$. There are few studies regarding reinfection rates in Spain. Ramos et al. (6) found a recrudescence rate of $4.7 \%$, and Gisbert et al. (7) recurrence rates of $3.6 \%$ at six months after eradication, $1.5 \%$ a year later, and $1.5 \%$ two years later, most of them recrudescences instead of true reinfections.

The main objectives of this study were to determine the annual reinfection rate within 4 years after successful treatment, and to evaluate the contribution of different factors to infection relapse.

\section{PATIENTS AND METHODS}

Two hundred and twenty-one patients successfully treated for $H$. pylori infection and under continuous monitoring in our GI Department were included. H. pylori infection 
eradication was diagnosed by using the urea breath test (UBT) or urease test, and histology in gastric ulcer patients.

Treatment indications were duodenal ulcer in 159 patients $(73 \%)$, dyspepsia in $30(12 \%)$, gastric ulcer in 18 $(9 \%)$, dermatological disease in $8(3 \%)$, and other in 6 patients $(3 \%)$. Sixty-one percent of patients were treated with proton pump inhibitors (PPI), clarithromycin, and amoxicillin; $16 \%$ with ranitidine bismuth citrate, and one or two antibiotics; 9\% with classic triple therapy (bismuth, ranitidine and metronidazole or tetracycline), and $14 \%$ with quadruple therapy and others.

Patients were monitored for recurrence with UBT annually for 4 years.

UBT was performed in patients after 6 hours fasting. After the administration of citric acid solutions ( $2 \mathrm{~g}$ of citric acid and $200 \mathrm{ml}$ of water) a baseline breath sample was taken. Then, $100 \mathrm{mg}$ of ${ }^{13} \mathrm{C}$-urea in $50 \mathrm{ml}$ of water were given, and 30 minutes later a second breath sample was taken. Samples were analyzed using mass spectrometry. Results (difference between both samples) were expressed in delta units, defined as the patient's ${ }^{13} \mathrm{C} /{ }^{12} \mathrm{C}$ ratio versus standard values expressed in percentage (\%o). Results were considered negative if they were under 5.

Patients were divided into two groups: reinfected, if a recurrence of $H$. pylori infection was observed by any test, and non-reinfected, if UBT was persistently negative. Age, gender, rural/urban environment, smoking habits, treatment regimens for $H$. pylori, diagnostic tests (UBT vs rapid urease test and histological examination), and UBT values were considered as predictors for $H$. pylori infection recurrence.

A multivariate logistic regression analysis was performed to evaluate the influence of variables in $H$. pylori infection recurrence. The Kaplan-Meier method was used to study the evolution of reinfection over time. The Local Ethics Committee approved the protocol study.

\section{RESULTS}

In all, 208 patients (121 males and 87 females, with a mean age of $50 \pm 13$ years, age range 18-81 years) completed the follow-up. Thirteen subjects $(6 \%)$ were excluded as they did not show up for any of the annual clinical visits.

H. pylori was found in 20 of 208 patients (incidence of 2.4 patients/year). Probability of reinfection according to the Kaplan-Meier method was estimated as 6.7\% (14/208 patients) in the first year, $1.9 \%(4 / 208)$ in the second year, $1 \%$ $(2 / 208)$ in the third, and $0 \%$ in the fourth year.

Reinfected patients were younger than non-reinfected patients (44.25 years vs 21.5 years, $\mathrm{p}=0.005)$. Age was considered a protecting factor in the multivariate analysis, with an odds ratio of 0.915 (95\% confidence interval, 0.803 to 0.979) (Table I).

The Kaplan-Meier test applied by age groups showed that the highest percentage of reinfection $(15.15 \%)$ occurred in subjects aged between 35 and 49; 65\% (13/20) of reinfected subjects were under 49 (Table II).

UBT test value after eradication in reinfected patients was higher than in non-reinfected patients (1.8 vs $0.4, \mathrm{p}=$ $0.042)$. In the logistics regression analysis UBT value after H. pylori treatment was shown to be a predictor for recurrence, with an odds ratio of 3.267 (95\% confidence interval, 0.684 to 15.599$)$.

No differences were found between both groups in gender, rural/urban environment, smoking habits, treatment regimens for $H$. pylori, or diagnostic tests used to confirm eradication. None of these variables were predictors of reinfection in the multivariate analysis.

During follow-up, one case of ulcer secondary to NSAID ingestion was diagnosed in the non-reinfected group. In the reinfected group 5 cases of duodenal ulcer and one of chronic urticaria relapse were diagnosed. All of them were treated successfully, and no ulcer complications were found.

Table I. Demographic and clinical characteristics of non-reinfected and reinfected patients

\begin{tabular}{|c|c|c|c|c|}
\hline Variables & & Non-reinfected & Reinfected & $p$ \\
\hline Mean age & & 51.50 years & 44.25 years & $0.005^{*}$ \\
\hline Gender & $\begin{array}{l}\text { Female } \\
\text { Male }\end{array}$ & $\begin{array}{l}42.5 \% \\
57.5 \%\end{array}$ & $\begin{array}{l}35 \% \\
65 \%\end{array}$ & 0.344 \\
\hline Environment & $\begin{array}{l}\text { Rural } \\
\text { Urban }\end{array}$ & $\begin{array}{l}49 \% \\
51 \%\end{array}$ & $\begin{array}{l}65 \% \\
35 \%\end{array}$ & 0.124 \\
\hline Smoking habit & & $11,7 \%$ & $10 \%$ & 0.503 \\
\hline Treatment regimens & $\begin{array}{l}\mathrm{PPI}+\mathrm{Amoxi}+\text { Clarithr } \\
\text { RBC+ATB } \\
\text { CTT } \\
\text { Other }\end{array}$ & $\begin{array}{c}62.8 \% \\
14.9 \% \\
8.5 \% \\
13.8 \%\end{array}$ & $\begin{array}{l}45 \% \\
25 \% \\
10 \% \\
20 \%\end{array}$ & 0.475 \\
\hline UBT mean values & & $0.44 \%$ \% units & $1.38 \%$ o $\delta$ units & 0.001 * \\
\hline
\end{tabular}

$\mathrm{PPI}+\mathrm{Amoxi}+$ Clarithr: proton pump inhibitors, amoxicillin, and clarithromycin

RBC+ATB: ranitidine bismuth citrate and one or two antibiotics.

CTT: classic triple therapy (bismuth, ranitidine and metronidazole or tetracycline).

*: Statistically significant. 
Table II. Reinfection rates by age group (Kaplan-Meier method)

\begin{tabular}{lcccc}
\hline Age groups & Patients (number) & Reinfected (number) & Reinfected (\%) & Cumulative reinfection (\%) \\
\hline$\leq 34$ years & 23 & 3 & $13.04 \%$ & $13.04 \%$ \\
$35-49$ years & 66 & 10 & $15.15 \% *$ & $14.60 \% * *$ \\
$50-64$ years & 88 & 7 & $7.95 \%$ & $11.29 \%$ \\
$\geq 65$ years & 31 & 0 & $0 \%$ & $9.61 \%$ \\
\hline
\end{tabular}

*: age group with the highest percentage of reinfection.

$* *$ : highest percentage of cumulative reinfection in subjects under 50 .

\section{DISCUSSION}

In our study, the recurrence rate after eradication of $H$. pylori infection was $2.4 \%$, similar to that in previous reports.

In a review that included countries with different levels of development, the annual incidence of recurrence was between 0 and $10 \%$ (8). Archimaditis et al. (9) found in 165 patients with duodenal ulcer a recrudescence rate of $9.7 \%$, and a reinfection rate of $9.3 \%$ in the second year, of $2.3 \%$ in the third year, and of $0 \%$ in the fourth (9). In Spain, Mones et al. (10) calculated a recurrence rate of $3.4 \%$ during the first year for 85 patients with peptic ulcer. In Brazil, Della Libera reported a reinfection rate of $7.6 \%$ (11) one year after successful eradication. In Japan, a country with a high prevalence of $H$. pylori infection, the annual reinfection rate was $0.8 \%$ (12). There are some studies that analyzed reinfection rates in patients with MALT lymphoma associated with $H$. pylori infection (13-15). One of these studies (16) observed a high recurrence rate (14\%) 22 months after the eradication therapy.

A detection of different $H$. pylori strains is necessary to differentiate between reinfection and recrudescence. Strains can be isolated using polymerase chain reaction (PCR) or restriction fragment length polymorphism (RFLP) analysis. However, it is controversial whether the definition requires the presence of similar or different strains with regard to the primary infection. Moreover, patients can be simultaneously infected by different strains (17-20). Since a genetic analysis was not performed in this study, we cannot distinguish between recrudescence and true reinfection.

Some factors have been proposed as adjuvant of infection recurrence, such as impaired immune response and low socioeconomic status (21). All patients in this study had the same socioeconomic status, so the influence of this factor has not been studied. We observed that reinfected patients were younger than non-reinfected ones, which is consistent with previous reports. $H$. pylori eradication at a young age can be a risk factor for infection recurrence $(22,23)$. Some studies found dental plaque (24-26) or partner infection (2729) was a potential risk factor for $H$. pylori recurrence.

Another controversial aspect is the clinical outcome of reinfection. Different situations may occur: infection relapse, symptomatic or not, without endoscopic findings; symptomatic ulcer relapse and its complications (mainly bleeding), and gastric MALT lymphoma recurrence after eradication success and histological improvement $(13,14)$.

It has been reported that ulcer relapse in reinfected pa- tients ranges from 3 to $60 \%$ (30-32); in this study we observed a rate of $20 \%$. Some studies demonstrated that eradication therapy is more effective than PPI treatment to prevent ulcer relapse and its complications (33-36). We could avoid them by using a therapy against $H$. pylori infection in reinfected patients.

However, management of reinfected patients remains unclear as yet. Some authors have questioned if $H$. pylori status needs to be periodically tested and treated in case of infection, above all in the case of MALT lymphoma or bleeding ulcer (37-39).

UBT should be the first-choice test for detecting $H$. $p y$ lori reinfection, since it is a non-invasive method that explores the whole stomach. Recently, a multicentric study published in our country has validated this test for the diagnosis and eradication control of $H$. pylori infection (40), since its sensitivity is high in both cases. However, in the second case, it is recommended that the cut-off value be lowered to 4.6 atoms per thousand. In fact, some authors establish their cut-off value in 3.5 atoms per thousand (41). In our study, if we had established it in 3.5 atoms per thousand, $15 \%(3 / 20)$ of reinfected patients would have been diagnosed with failed eradication instead of infection recurrence. No differences were observed in the non-reinfected group. Therefore, values between 3.5 and 5 atoms per thousand should be considered indecisive, so another UBT, histological studies or rapid urease test should be performed.

In order to detect a recurrence of $H$. pylori infection and to retreat patients to prevent complications, we suggest an annual follow-up with UBT for patients under 50 who are asymptomatic and were successfully treated.

In conclusion, in our population the $H$. pylori recurrence rate is 2.4 patients/year within a 4-year follow-up period. This study has shown that reinfection depends on age, so we recommend an annual UBT for 3 years after eradication, above all in patients under 50 years of age.

\section{REFERENCES}

1. Malfertheiner P, Leodolter A, Peitz U. Cure of Helicobacter pyloriassociated ulcer disease through eradication. Baillieres Best Pract Res Clin Gastroenterol 2000; 14 (1): 119-32.

2. Gisbert JP. Recurrencia de la infección por Helicobacter pylori. En: Pajares García JM, Correa P, Pérez Pérez GI, eds. Infección por Helicobacter pylori en lesiones gastroduodenales. La segunda década. Barcelona: Prous Science, 1998. p. 263-78.

3. Labenz J. Consequences of Helicobacter pylori cure in ulcer patients. Baillieres Best Pract Res Clin Gastroenterol 2000; 14 (1): 133-45. 
4. Khor CJ, Fock KM, Ng TM, et al. Recurrence of Helicobacter pylori infection and duodenal ulcer relapse, following successful eradication in an urban east Asian population. Singapore Med J 2000; 41 (8): 382-6.

5. Carta M, Dore MP, Idda M, et al. Effect of cure rate on reinfection with $H$. pylori: a three-year, follow-up study. Am J Gastroenterol 2000; 95 (11): 3324-5.

6. Ramos M, Pallarés H, Garrido M. Eficacia, grado de cicatrización resistencia in vitro y tasa de reinfección anual después del tratamiento erradicador del Helicobacter pylori en nuestra área. Rev Esp Enferm Dig 1997; 89 (Supl. 1): 77.

7. Gisbert JP, Pajares JM, García-Valriberas R, et al. Recurrence of Helicobacter pylori infection after eradication. Incidence and variables influencing it. Scand J Gastroenterol 1998; 33: 1144-51.

8. Gisbert JP. Reinfección por Helicobacter pylori tras su erradicación: ¿un temor infundado? Med Clin 1998; 111: 380-4.

9. Archimeditis A, Balastos V, Delis V, et al. Reappearance of Helicobacter pylori after eradication: Implications on duodenal ulcer recurrence. A prospective 6 year study. J Clin Gastroenterol 1999; 28 (4): 345-7.

10. Monés J, Rodrigo L, Sancho F, et al. Erradicación de Helicobacter pylori versus tratamiento de mantenimiento durante un año: eficacia sobre la recidiva y la gastritis. Rev Esp Enferm Dig 2001; 93 (6): 372-80.

11. Della Libera E, Rohr MRS, Moraes M, et al. Eradication of Helicobacter pylori infection in patients with duodenal ulcer and non-ulcer dyspepsia and analysis of one-year reinfection rates. Braz J Med Biol Res 2001; 34: 753-7.

12. Adachi M, Mizuno M, Yokota K, et al. Reinfection rate following effective therapy against Helicobacter pylori infection in Japan. J Gastroenterol Hepatol 2002; 17 (1): 27-31.

13. Pellicano R, Peyre S, Leone N, et al. The effect of the eradication of Helicobacter pylori infection on hemorrhage because of duodenal ulcer. J Clin Gastroenterol 2001; 32 (3): 222-4.

14. Fischbach W, Jung T, Goebeler-Kolve M, Eck M. Comparative analysis of the Helicobacter pylori status in patients with gastric MALT-type lymphoma and their respective spouses. Z Gastroenterol 2000; 38 (8): 627-30.

15. Haruma K. Trend toward a reduced prevalence of Helicobacter pylori infection, chronic gastritis, and gastric cancer in Japan. Gastroenterol Clin North Am 2000; 29 (3): 623-32.

16. Papa A, Cammarota G, Tursi A, Gasbarrini A, Gasbarrini G. Helicobacter pylori eradication and remission of low-grade gastric mucosaassociated lymphoid tissue lymphoma: a long-term follow-up study. J Clin Gastroenterol 2000; 31 (2): 169-71.

17. Jeen YT, Lee SW, Kwon SI, et al. Differentiation between reinfection and recrudescence of Helicobacter pylori strains using PCR-based restriction fragment length polymorphism analysis. Yonsei Med J 2001; 42 (1): 41-5.

18. Morgner A, Lehn N, Andersen LP, et al. Helicobacter heilmannii-associated primary gastric low-grade MALT lymphoma: complete remission after curing the infection. Gastroenterology 2000; 118 (5): 821-8.

19. Simsek IS, Menevse S, Sahin FI. PCR and RFLP analysis for identification and typing of Helicobacter pylori strains isolated from gastric biopsy specimens. Tohoku J Exp Med 2000; 190 (3): 21322.

20. Neiger R, Simpson KW. Helicobacter infection in dogs and cats: facts and fiction. J Vet Intern Med 2000; 14 (2): 125-33.

21. Aydin A, Ersoz G, Ozutemiz O, Tuncyurek M. Low reinfection rate of Helicobacter pylori infection in Turkey. J Clin Gastroenterol 2000; 30 (3): 337
22. Shimizu T, Yarita Y, Kaneko K, et al. Case of intrafamilial Helicobacter pylori reinfection after successful eradication therapy. Pediatr Infect Dis J 2000; 19 (9): 901-3.

23. Sung JJ. Where are we with current therapy? Helicobacter 2000; 5 (Supl. 1): S17-21; discussion S27-31.

24. Gurbuz AK, Ozel AM, Yazgan Y, et al. Oral colonization of Helicobacter pylori: risk factors and response to eradication therapy. South Med J 2003; 96 (3): 244-7.

25. Karczewska E, Konturek JE, Konturek PC, et al. Oral cavity as a potential source of gastric reinfection by Helicobacter pylori. Dig Dis Sci 2002; 47 (5): 978-86.

26. Kilmartin CM. Dental implications of Helicobacter pylori. J Can Dent Assoc 2002; 68 (8): 489-93.

27. Gisbert JP, Boixeda D. Papel de la infección del cónyuge en las reinfecciones tras la erradicación de Helicobacter pylori. Rev Esp Enferm Dig 1998; 110: 56-61.

28. Gisbert JP, García-Aranda I, Boixeda D, Barba M, Cantón R, García Plaza A, et al. Role of partner's infection in reinfections after $H$. pylo$r i$ eradication. Eur J Gastroenterol Hepatol 2002; 14: 865-71.

29. Deltenre M, de Koster E. How come I've got it? (A review of Helicobacter pylori transmission). Eur J Gastroenterol Hepatol 2000; 12 (5): 479-82.

30. Bapat MR, Abraham P, Bhandarkar PV, Phadke Ay, Joshi AS. Acquisition of Helicobacter pylori infection and reinfection after its eradication are uncommon in Indian adults. Indian J Gastroenterol 2000 19 (4): 172-4

31. Fujioka T, Uribe RU, Kubota T. Peptic ulcer recurrence after Helicobacter pylori eradication: A 5-year follow-up study. Eur J Gastroenterol Hepatol 1995; 7 (Supl. 1): 35-8.

32. Hildebrand P, Bardhan P, Rossi L, et al. Recrudescence and reinfection with Helicobacter pylori after eradication therapy in Bangladeshi adults. Gastroenterology 2001; 121 (4): 792-8.

33. Chen T, Chang F, Lee S, et al. Recurrence of $H$. pylori infection and dyspeptic symptoms after successful eradication in patients cured of duodenal ulcer disease. Hepato-Gastroenterology 1999; 46 (25): 252

34. Axon ATR, O'Morain CA, Bardhan KD, et al. Randomised double blind controlled study of recurrence of gastric ulcer after treatment for eradication Helicobacter pylori infection. Br Med J 1997; 314 (7080): $565-8$

35. Van der Hulst RWM, Rauws EAJ, Koyan B. Prevention of ulcer recurrence after eradication Helicobacter pylori: a prospective long-term follow-up study. Gastroenterology 1997; 113 (4): 1082-6.

36. Borody TJ, Andrews P. Helicobacter pylori reinfection rate in patients with cured duodenal ulcer. Am J Gastroenterol 1994; 89: 52932.

37. Seo M, Okada M, Shirotani T, et al. Recurrence of Helicobacter pylo$r i$ infection and the long-term outcome of peptic ulcer after successful eradication in Japan. J Clin Gastroenterol 2002; 34 (2): 129-34.

38. Miehlke S, Bayerdörffer E, Lehn N, et al. Recurrence of duodenal ulcers during five years of follow-up after cure of Helicobacter pylor infection. Eur J Gastroenterol Hepatol 1995; 7: 975-8.

39. Horstmann M, Erttmann R. Relapse of MALT lynphoma associated with Helicobacter pylori after antibiotic treatment. Lancet 1994; 343 1098-9.

40. Gisbert JP, Ducons J, Gomollon F, et al. Validación de la prueba del aliento con ${ }^{13} \mathrm{C}$-urea para el diagnóstico inicial de la infección por $\mathrm{He}$ licobacter pylori y la confirmación de su erradicación tras el tratamiento. Rev Esp Enferm Dig 2003; 95 (2): 115-20.

41. Johnston BJ, Levi S, Johnston PG. Cut-off point for ${ }^{13} \mathrm{C}$-urea breath test. Gut 1996; 39 (Supl. 2): A 122. 


\title{
Incidencia y variables que influyen en la recurrencia de infección por Helicobacter pylori
}

\author{
B. J. Gómez Rodríguez, M. Rojas Feria, M. J. García Montes, R. Romero Castro, P. Hergueta Delgado, \\ F. J. Pellicer Bautista y J. M. Herrerías Gutiérrez
}

Servicio de Aparato Digestivo. Hospital Universitario Virgen Macarena. Sevilla

\section{RESUMEN}

Objetivos: a) determinar la frecuencia de reinfección por $H$. pylori durante un seguimiento de cuatro años tras la erradicación; y b) evaluar la influencia de distintos factores en la aparición de dicha recurrencia.

Pacientes y métodos: se llevó a cabo el estudio en 208 pacientes, 121 varones y 87 mujeres; con una edad media de 50 años y un intervalo entre 18 y 81 años. Los pacientes habían sido tratados previamente y en todos ellos se comprobó la eficacia de la erradicación. Para determinar las tasas de recurrencia, se comprobó el status de $H$. pylori mediante test de aliento posterradicación anualmente durante cuatro años. Como posibles factores relacionados con la recurrencia de la infección se consideraron la edad, el sexo, el hábitat rural o urbano, el tabaquismo, el régimen terapéutico utilizado, el método de comprobación de la erradicación y el valor de la prueba del aliento (\%o unidades $\delta$ ) al mes de completar la terapia.

Resultados: se reinfectaron un 9,6\% de los pacientes observados durante el seguimiento. En el primer año posterradicación sufrieron reinfección el 6,7\% (14/208 pacientes), en el segundo el 1,9\% (4/208), en el tercero el 1\% (2/208) y en el cuarto el $0 \%$. En el análisis multivariante, resultaron factores de riesgo la menor edad de los pacientes y los valores elevados del test de aliento.

Conclusiones: la tasa de reinfección anual fue de 2,4 pacientes-año. La menor edad de los pacientes y un valor más elevado en el test de aliento posterradicación serían factores predictivos de reinfección. Sería recomendable un seguimiento con pruebas de aliento anuales, sobre todo, en menores de 50 años, para la detección de reinfección y así evitar complicaciones ulcerosas ulteriores.

Palabras clave: Reinfección. Recrudescencia. Recurrencia. Erradicación. Helicobacter pylori. Test de aliento.

\section{INTRODUCCIÓN Y OBJETIVOS}

La infección por Helicobacter pylori es un factor esencial en la patogénesis de la enfermedad ulcerosa péptica, el linfoma MALT y el adenocarcinoma gástricos. La erradicación de la bacteria cura la úlcera activa y, a largo plazo, da lugar a una drástica reducción de la reaparición de la úlcera y sus complicaciones (1).

Existen dos tipos de recurrencia de la infección por $H$. pylori, una vez erradicada la bacteria tras un tratamiento efectivo. Un mecanismo sería la recrudescencia, definida como la detección de una cepa de la bacteria similar a la aislada previamente a la erradicación. El otro mecanismo es la verdadera reinfección, esto es, cuando el paciente es infectado por una cepa distinta de H. pylori $(2,3)$.

La tasa de reinfección se sitúa en países subdesarrollados entre el 6,25\% en algunas zonas de la población asiática (4) y el 2,4\% en ciertas áreas de la población india (5). Por el contrario, la tasa en países desarrollados es inferior al $1 \%$. En España existen pocos estudios sobre reinfección, destacando el de Ramos y cols. (6) que halla una tasa de recrudescencia de $4,7 \%$ y el de Gisbert y cols., que encuentra tasas de recurrencia del 3,6\% a los 6 meses, $1,5 \%$ al año y $1,5 \%$ a los 2 años, representando la mayoría recrudescencias en lugar de verdaderas reinfecciones (7).

Los objetivos fundamentales en este estudio fueron en primer lugar, determinar la tasa anual de reinfección por $H$. pylori hasta cuatro años después de haber realizado tratamiento erradicador efectivo contra la bacteria. En segundo término, evaluar la influencia de distintos factores en la reaparición de infección por el microorganismo.

\section{PACIENTES Y MÉTODOS}

Se incluyeron 221 pacientes erradicados, que acudían a revisión a consultas de Digestivo, siendo el método diagnóstico de curación de la infección la negatividad del test de aliento con ${ }^{13} \mathrm{C}$-urea, excepto en los pacientes con úlcera gástrica, en los que se tomaron múltiples biopsias de antro y cuerpo para test de la ureasa rápida y estudio histológico que debían ser ambos negativos.

Las indicaciones del tratamiento frente a la infección por $H$. pylori fueron: ulcus duodenal en 159 pacientes (73\%); dispepsia en 30 (12\%); ulcus gástrico en 18 (9\%); manifestaciones cutáneas en 8 (3\%); y otras causas en 6 (3\%). Las pautas de tratamiento empleadas fueron: inhibidor de bomba de protones, claritromicina y amoxicilina en el $61 \%$ de los pacientes; ranitidina citrato de bismuto con uno o dos antibióticos en el $16 \%$ de los casos, triple terapia clásica (bismuto, ranitidina y metronidazol o tetraciclinas) en el $9 \%$; y cuádruples terapias y otras en el $14 \%$ del total.

Para determinar las tasas de recurrencia de la infección se realizó un seguimiento anual de los pacientes durante 4 años, 
comprobando el status de $H$. pylori mediante la realización de test de aliento con ${ }^{13} \mathrm{C}$-urea.

La realización de la prueba del aliento se hacía en ayunas de 6 horas, administrándose inicialmente una solución de 200 $\mathrm{ml}$ de agua con 2 gramos de ácido cítrico, y recogiéndose una muestra basal de aliento. Inmediatamente después el paciente ingería una solución de $100 \mathrm{mg}$ de ${ }^{13} \mathrm{C}$-urea en $50 \mathrm{ml}$ de agua. Tras unos 30 minutos se procedía a recoger una segunda muestra postdosis de aliento. Ambas muestras eran analizadas mediante un espectrómetro de masas, siendo la diferencia entre ambas determinaciones el resultado del test, emitido en unidades $\delta$, definida como la expresión en tantos por mil de la relación de ${ }^{13} \mathrm{C} /{ }^{12} \mathrm{C}$ del paciente respecto del estándar. Como punto de corte para una interpretación negativa de la prueba se estableció un valor inferior a 5 átomos por mil.

Se agruparon a los pacientes en: reinfectados, en los que se detectaba recurrencia de infección cuando alguna de las pruebas era positiva; y no reinfectados, cuando todos los tests de aliento durante el seguimiento eran negativos.

Como posibles factores predictivos de recurrencia de la infección por $H$. pylori se consideraron: la edad, el sexo, el hábitat rural o urbano, el tabaquismo, el tipo de terapia erradicadora usada, los métodos diagnósticos para confirmar la erradicación (prueba del aliento frente a histología más prueba de ureasa rápida) y el valor medio en cada grupo del test de aliento al mes de la erradicación.

A continuación se llevó a cabo un estudio estadístico multivariante, mediante un modelo de regresión logística no condicional para evaluar la influencia de las variables estudiadas en la recurrencia de infección por H. pylori. Además se utilizó el método de Kaplan y Meier para estudiar la evolución temporal de las reinfecciones.

El protocolo de este estudio fue aprobado por el Comité Ético de Investigación Clínica del Área Hospitalaria correspondiente, ajustándose a las normas éticas internacionales.

\section{RESULTADOS}

Del total de los pacientes incluidos completaron el seguimiento 208 pacientes, 121 varones y 87 mujeres; con una edad media de $50 \pm 13$ años y un intervalo de 18 y 81 años. Se perdieron 13 sujetos $(6 \%)$ que no acudieron a alguna de las revisiones anuales, por lo que fueron excluidos del estudio.

Durante el seguimiento posterradicación se hallaron recurrencias en 20 de los 208 pacientes, lo que representa una incidencia de 2,4 pacientes-año.

Según el método de Kaplan y Meier la probabilidad en el tiempo de sufrir reinfecciones en el primer año posterradicación fue del 6,7\% (14/208 pacientes), en el segundo del $1,9 \%(4 / 208)$, en el tercero del $1 \%(2 / 208)$ y en el cuarto del $0 \%$.

El grupo de pacientes reinfectados era más joven que los que no sufrieron recurrencia de la infección (promedio de edad: 44,25 años frente a 51,5 años, p=0,005). El aumento en la edad de los pacientes resultó ser un factor protector frente a la recurrencia (Tabla I) en el estudio multivariante y mostró una Odds Ratio de 0,915 (intervalo de confianza del $95 \%$ entre 0,803 y 0,979 ).

Según el método de Kaplan y Meier aplicado sobre grupos de edad predefinidos (Tabla II), se halló que el mayor porcentaje de reinfecciones $(15,15 \%)$ ocurrió en los sujetos con edades comprendidas entre los 35 y 49 años. El $65 \%$ de reinfectados (13/20) tenían una edad inferior a 49 años.

El valor promedio del test de aliento posterradicación en reinfectados fue superior al de no reinfectados (1,8 frente a $0,4 ; p=0,042)$. En la regresión logística el valor de la prueba del aliento tras la terapia erradicadora se mostró como factor predictivo de recurrencia de infección con una $O d d s$ Ratio de 3,267 (intervalo de confianza del $95 \%$ entre 0,684 y 15,599).

Tabla I. Comparación de las distintas variables estudiadas entre el grupo de reinfectados y no reinfectados

\begin{tabular}{|c|c|c|c|c|}
\hline Variables & & No reinfectados & Reinfectados & $p$ \\
\hline Promedio Edad & & 51,50 años & 44,25 años & $0,005^{*}$ \\
\hline Sexo & $\begin{array}{l}\text { Mujeres } \\
\text { Hombres }\end{array}$ & $\begin{array}{l}42,5 \% \\
57,5 \%\end{array}$ & $\begin{array}{l}35 \% \\
65 \%\end{array}$ & 0,344 \\
\hline Hábitat & $\begin{array}{l}\text { Rural } \\
\text { Urbano }\end{array}$ & $\begin{array}{l}49 \% \\
51 \%\end{array}$ & $\begin{array}{l}65 \% \\
35 \%\end{array}$ & 0,124 \\
\hline Tabaquismo & & $11,7 \%$ & $10 \%$ & 0,503 \\
\hline Reg. Terapéutico & $\begin{array}{l}\text { IBP+Amoxi+Claritro } \\
\text { RCB+ATBs } \\
\text { TTC } \\
\text { Otras terapias }\end{array}$ & $\begin{array}{c}62,8 \% \\
14,9 \% \\
8,5 \% \\
13,8 \%\end{array}$ & $\begin{array}{l}45 \% \\
25 \% \\
10 \% \\
20 \%\end{array}$ & 0,475 \\
\hline Valor Medio Aliento & & $0,44 \%$ unid. $\delta$ & $1,38 \%$ unid. $\delta$ & 0,001 * \\
\hline
\end{tabular}

Diagn Erradicación: método usado para comprobar la erradicación.

Reg terapéutico: terapia erradicadora utilizada.

IBP+Amoxi+Claritro: inhibidores de la bomba de protones con amoxicilina y claritromicina.

RCB+ATBs: ranitidina citrato de bismuto con uno o dos antibióticos.

TTC: triple terapia clásica (bismuto con ranitidina con tetraciclinas o metronidazol).

Valor Medio Aliento: valor promedio en cada grupo de la prueba del aliento de comprobación de la erradicación expresado en \%o unidades $\delta$

*: Significación estadística. 
Tabla II. Frecuencias de reinfección según grupos etarios (método de Kaplan y Meier)

\begin{tabular}{lcccc}
\hline Grupos etarios & $N^{\circ}$ Pacientes & $N^{\circ}$ Reinfectados & Reinfección & Reinfección Acumulada \\
\hline$\leq 34$ años & 23 & 3 & $13,04 \%$ & $13,04 \%$ \\
$35-49$ años & 66 & 10 & $15,15 \% *$ & $14,60 \% * *$ \\
$50-64$ años & 88 & 7 & $7,95 \%$ & $11,29 \%$ \\
$\geq 65$ años & 31 & 0 & $0 \%$ & $9,61 \%$ \\
\hline
\end{tabular}

*: grupo etario con mayor frecuencia de reinfecciones.

**: mayor porcentaje de reinfección acumulada en $<50$ años.

No se han apreciado diferencias entre ambos grupos en el sexo, hábitat rural o urbano, hábito tabáquico de los sujetos, régimen erradicador empleado y método de confirmación de la erradicación. Según el estudio multivariante ninguna de estas variables es predictiva de reinfección.

Durante el seguimiento se diagnosticó un caso de ulcus secundario a la ingesta reciente de antiinflamatorios no esteroideos en el grupo de los no reinfectados frente a cinco casos de úlcera duodenal y un caso de reaparición de urticaria crónica en el grupo de los reinfectados. Todos estos pacientes fueron retratados con éxito, sin que llegaran a producirse complicaciones ulcerosas.

\section{DISCUSIÓN}

La recurrencia de infección por $H$. pylori hallada en este estudio es del 2,4 pacientes-año, acorde con las publicadas previamente.

En una revisión que incluye a países con distinto nivel desarrollo, se observa una incidencia anual entre el 0 y el $10 \%$ (8). Archimaditis y cols. estudian a 165 pacientes con ulcus duodenal siendo la recrudescencia del 9,7\%, la reinfección al segundo año del 9,3\%, al tercer año del $2,3 \%$ y del $0 \%$ a partir del cuarto año (9). En España, Monés y cols. en un estudio sobre 85 enfermos con úlcera péptica obtienen una cifra de recurrencia al año del 3,4\% (10). Della Libera halla en Brasil una tasa de reinfección en el primer año tras la erradicación del 7,6\% (11). En Japón, donde existe una alta prevalencia de infección por $H$. pylori, se han publicado cifras de reinfección anual del $0,8 \%$ (12). Existen distintos trabajos que analizan la reinfección sobre enfermos erradicados por MALT gástrico $(13,14,15)$; uno de los cuáles obtiene unas tasas elevadas a los 22 meses (14\%) (16).

Para diferenciar recrudescencia de reinfección se requiere la detección de distintas cepas bacterianas, bien mediante la reacción en cadena de la polimerasa (PCR) o el análisis de polimorfismos genéticos (RFLP). No obstante, existe controversia en si la definición de reinfección requiere que la cepa reinfectante sea la misma u otra diferente de la inicial. Más aún, el paciente podría estar previamente infectado por varias cepas de forma simultánea. (17-20). En este estudio no se han realizado técnicas genéticas, por lo que no podemos conocer la cepa infectante ni diferenciar aquellos pacientes que han sufrido recrudescencia o reinfección verdadera.
Se han propuesto distintos factores como coadyuvantes de la recurrencia de la infección, como la falta de inmunidad natural para la reinfección o el bajo nivel socioeconómico (21). La totalidad de los pacientes de la nuestra serie pertenecían a un nivel medio, por lo que no hemos estudiado la influencia de este factor. En nuestro estudio los pacientes reinfectados tenían una menor edad, tal y como se ha publicado anteriormente, llegando a constituir un factor de riesgo la erradicación a edades tempranas $(22,23)$. Otros trabajos citan como factores de riesgo la presencia de $H$. pylori en la placa dental (24-26), o el reservorio de la infección en el ámbito familiar (27-29).

Otro aspecto motivo de controversia son las implicaciones clínicas de la reinfección por $H$. pylori. Tras la reinfección pueden ocurrir diversas circunstancias: la reaparición de la infección con o sin síntomas pero sin lesiones, la recidiva ulcerosa con reaparición de la clínica y complicaciones (fundamentalmente con sangrado), y la recidiva de linfoma MALT gástrico, tras haber regresado histológicamente después de la erradicación de $H$. pylori $(13,14)$.

La recurrencia ulcerosa en pacientes reinfectados oscila en distintas series entre el 3 y el $60 \%$ (30-32), siendo en el presente estudio del $20 \%$. La mayoría de los trabajos demuestran, además, que la erradicación es más efectiva que el tratamiento con antisecretores de mantenimiento para prevenir recidivas y complicaciones ulcerosas. En nuestra serie se logró evitar dichas complicaciones retratando a los pacientes reinfectados (33-36).

Todos estos aspectos plantean dudas sobre el manejo de los sujetos reinfectados, preguntándose algunos autores si debería seguirse una comprobación periódica y mantenida del status de infección por $H$. pylori en estos pacientes y una nueva erradicación cuando estuviera indicado clínicamente, sobre todo, en casos de linfoma MALT o hemorragia ulcerosa (37-39).

La prueba del aliento debería ser la prueba diagnóstica de primera elección para la detección de reinfección, por su nula invasividad y capacidad para explorar toda la cavidad gástrica. En un ensayo clínico multicéntrico publicado recientemente en nuestro país se ha validado esta técnica en el diagnóstico de la infección de $H$. pylori y el control de su erradicación (40), ofreciendo una alta sensibilidad en ambos casos, si bien en la segunda indicación se aconseja bajar el punto de corte a 4,6 átomos por mil. Otros autores lo bajan incluso hasta el 3,5 átomos por mil (41). En nuestra experiencia, si el nivel de corte de interpretación se hubiese baja- 
do a $3,5 \%$, se hubieran detectado hasta un $15 \%$ de pacientes $(3 / 20$ reinfectados) en los que posteriormente se detectó reinfección, y que por lo tanto, se hubieran considerado como no erradicados al inicio del seguimiento. En el grupo de no reinfectados no se detectaba ningún sujeto al bajar el punto de corte. Por ello, un resultado negativo de esta prueba con un valor entre 3,5 y $5 \%$ debe ser valorado con prudencia (7), e incluso debería plantearse repetir la prueba o compararla con otros métodos como la histología o el test de ureasa rápida.

Atendiendo a nuestros resultados, se podría proponer en aquellos pacientes erradicados y asintomáticos, especialmente menores de 50 años, el seguimiento mediante prueba de aliento anual, durante los tres primeros años, para detectar una posible reinfección, que se beneficiara de un retratamiento, antes de la aparición de complicaciones. En cambio, en los pacientes erradicados sintomáticos se debería realizar endoscopia digestiva alta diagnóstica.

Como conclusión, la tasa de reinfección por $H$. pylori en nuestro medio tras 4 años de seguimiento fue de 2,4 pacientes-año. Parece ser que la menor edad de los pacientes favorece la reaparición de la infección, por lo que consideramos conveniente un seguimiento anual mediante prueba del aliento durante los tres primeros años tras la erradicación, sobre todo en pacientes menores de 50 años. 